\title{
Evidências Adicionais de Validade da Escala de Satisfação no Trabalho
}

\author{
Luara Carvalho ${ }^{1}$ D \\ Universidade Salgado de Oliveira, Niterói-RJ, Brasil \\ Hudson Golino (D) \\ University of Virginia, Virgínia, Estados Unidos \\ Luciana Mourão (iD) \\ Universidade Salgado de Oliveira, Niterói-RJ, Brasil
}

\section{RESUMO}

Este estudo objetivou identificar as propriedades da escala de satisfação no trabalho mais utilizada em amostras brasileiras em termos de sua estrutura, invariância da medida e validade convergente. Participaram da pesquisa 733 trabalhadores (46\% mulheres) do setor industrial e terciário de dois estados brasileiros. Além do modelo original de cinco fatores correlacionados, foram comparados três modelos estruturais alternativos (cinco fatores não correlacionados, hierárquico e bifatorial). Os resultados apontam que a estrutura bifatorial com cinco variáveis latentes de primeiro nível mais uma variável geral também de primeiro nível é um modelo estrutural robusto para avaliar a satisfação de trabalhadores brasileiros e foi invariante para todos os grupos testados, tendo a possibilidade de aplicação em amostras com diversidade em termos de tempo de trabalho, grau de escolaridade e sexo. A medida também apresentou correlação positiva de moderada a elevada com outras duas variáveis do comportamento organizacional, confirmando a validade convergente.

Palavras-chave: satisfação no trabalho; validade estatística; modelo bifatorial.

\section{ABSTRACT - Additional Validity Evidence for the Job Satisfaction Scale}

This study aimed to identify the properties of the job satisfaction scale most used in Brazilian samples in terms of its structure, convergent validity and measure invariance. The study involved 733 workers ( $46 \%$ women) from the industrial and tertiary sectors of two Brazilian states. In addition to the original model of five correlated factors, three alternative structural models were compared (five uncorrelated factors, hierarchical and bifactorial). The results show that the bifactorial structure, with five latent variables of the first level plus a general variable also of the first level, is a robust structural model to evaluate the satisfaction of Brazilian workers and was invariant for all groups tested, with the possibility of application in samples with different work times, levels of education and gender. The measure also showed a positive moderate to high correlation with the other two variables of organizational behavior confirming the convergent validity.

Keywords: job satisfaction, statistical validity; bifactor model.

\section{RESUMEN - Evidencias Adicionales de Validez de la Escala de Satisfacción Laboral}

Este estudio tuvo como objetivo identificar las propiedades de la escala de satisfacción laboral más utilizada en muestras brasileñas en términos de su estructura, validez convergente e invarianza de medida. Participaron de la encuesta 733 trabajadores (46\% mujeres) de los sectores industrial y terciario de dos provincias brasileñas. Además del modelo original de cinco factores correlacionados, se compararon tres modelos estructurales alternativos (cinco factores no correlacionados, jerárquico y el de dos factores). Los resultados demuestran que, la estructura de dos factores con cinco variables latentes del primer nivel más una variable general, también del primer nivel, es un modelo estructural robusto para evaluar la satisfacción laboral de los trabajadores brasileños y fue invariante para todos los grupos evaluados, con la posibilidad de aplicación en muestras con diferentes tiempos de trabajo, escolaridad y género. La medida también presentó correlación positiva, de moderada a elevada, con otras dos variables del comportamiento organizacional, lo que confirma la validez convergente.

Palabras clave: satisfacción laboral; validez estadística; modelo de dos factores.

A satisfação no trabalho é um fenômeno psicológico e compreende as relações entre indivíduo-trabalho e o impacto que elas podem gerar em termos de resultados individuais (van Vianen, 2018). Essa temática é amplamente pesquisada na Psicologia do Trabalho e das
Organizações - POT (Hora et al., 2018), figurando entre os 15 temas mais investigados na literatura nacional de comportamento organizacional desde 1996 (Mourão et al., 2016). Assim, embora o interesse sobre a satisfação no trabalho seja antigo (Locke, 1976; O'Reilly, 1991), ele

${ }^{1}$ Endereço para correspondência: Universidade Salgado de Oliveira (UNIVERSO), Programa de Pós-Graduação em Psicologia. Rua Marechal Deodoro, 263, Bloco A, Centro, 24020-420, Niterói, RJ. Tel.: (21) 96498-4492. E-mail: luaracarvalhomotta@gmail.com

Artigo derivado da Dissertação de mestrado de Luara Carvalho com orientação de Hudson Golino, defendida em 2018 no programa de pós-graduação em Psicologia da Universidade Salgado de Oliveira. 
continua crescente na POT (Farina et al., 2019; Hora et al., 2018) e em outras áreas, como saúde (Moura et al., 2020; Yasin et al., 2020) e educação (Lorenzo et al., 2020; Nascimento et al., 2019).

$\mathrm{Na}$ literatura internacional, o construto também mantém relevância com pesquisas sobre o tema em mais de 40 países e com mais de 60 escalas para a sua mensuração (Hora et al., 2018). O fato de haver tantas escalas sobre satisfação no trabalho nos permite questionar se as medidas utilizadas apresentam adequadas propriedades psicométricas. A revisão realizada por Hora et al. (2018) aponta que a Escala de Satisfação no Trabalho (EST) de Siqueira (2008) é a mais utilizada no Brasil, o segundo país do mundo com mais pesquisas sobre a temática. Além do destaque da satisfação no trabalho nas publicações nacionais e estrangeiras, do ponto de vista social, a temática é igualmente relevante. Isso fica evidenciado pelo crescente número de empresas que implementam programas voltados à satisfação de seus trabalhadores, inclusive como forma de gerar um ambiente laboral mais saudável.

Assim, nossa proposta é investigar a EST, de forma a tornar as pesquisas sobre a satisfação laboral mais robustas, contribuindo para a melhoria da qualidade das investigações no contexto acadêmico e social. Para tanto, discutiremos a estrutura fatorial dessa medida e realizaremos outros testes relativos à sua invariância em múltiplos grupos, bem como a possibilidade de convergência dessa medida com outros construtos teoricamente relacionados à satisfação no trabalho.

A importância de testar a estrutura da EST também se apoia no fato de haver uma confusão e fragmentação conceitual em torno do construto. Um exemplo são os artigos de bem-estar no trabalho, que com frequência pesquisam a satisfação, mas se intitulam como bem-estar (Hackman \& Oldham, 1975; Silva \& Ferreira, 2013). Tal fragmentação enreda o avanço científico, pois leva a construção de microteorias, resultando em um distanciamento dos conceitos de sua origem teórica e na dificuldade de muitos pesquisadores em delimitar os construtos que estão estudando (Borges-Andrade \& Zanelli, 2014). O chamado alargamento conceitual (Rodrigues \& Carvalho-Freitas, 2016), resulta então em uma proliferação também de escalas com baixa possibilidade de discriminação de seus itens.

Assim, é relevante discutir uma medida adequada para avaliar a satisfação em trabalhadores brasileiros que seja consonante com um conceito, modelo teórico, e com apropriados indicadores psicométricos. Uma das consequências da dificuldade de delimitar o conceito de satisfação no trabalho é o enfraquecimento da qualidade psicométrica dos instrumentos de avaliação, que muitas vezes deixam de lado a robustez teórica que deveria embasá-los. Muitas pesquisas que investigam a satisfação não deixam clara a definição adotada, o que dificulta a comparação dos resultados, tendo em vista a divergência das teorias que orientam as medidas (Hora et al., 2018; Marqueze \& Moreno, 2005; Staw, 1984).

A elaboração de uma definição geral de satisfação no trabalho, portanto, é uma tarefa árdua por três razões principais: 1. a amplitude pode derivar do interesse que o tema desperta, não só aos pesquisadores da área, mas aos profissionais alocados em cargos de nível gerencial (Judge et al., 2001); 2. a natureza histórica do fenômeno, uma vez que o próprio conceito de trabalho e carreira muda ao longo das décadas (Hall et al., 2018; Martins \& Santos, 2006); e 3. a questão transcultural, decorrente da diversidade de características de cada cultura e dos modos de relação com o trabalho que cada uma delas desenvolve (International Test Comission [ITC], 2018).

Nesse sentido, cabe apontar a multiplicidade teórica que circunda o construto. No final da década de 1940, a definição de satisfação aproximava-se do conceito de motivação, que foi uma junção comum até meados da década de 1970, mas que tem sido superada, uma vez que são construtos distintos (Martins \& Santos, 2006). A motivação é uma variável subjetiva que precede comportamentos diversos, entre eles a satisfação no trabalho. Embora a satisfação também seja uma avaliação subjetiva, envolve aspectos mais concretos, como o relacionamento com os colegas e a chefia, a identificação com a atividade realizada, a percepção de possibilidade de progresso e a avaliação sobre o salário (Siqueira, 2008).

A primeira teoria proposta para explicar o construto foi a Teoria dos Dois Fatores (Herzberg et al., 1959), que propõe a existência de duas dimensões separadas, uma relativa à satisfação e outra à insatisfação. Locke (1976), ao contrário, considera que a satisfação e a insatisfação no trabalho fazem parte de um mesmo fenômeno, não sendo aspectos dissociados. Alguns autores corroboram essa ideia de que ambas estão em um contínuo (Begley \& Czajka, 1993), embora outros concordem com a teoria de Herzberg et al. (1959), que a divide em dois construtos (Martins \& Santos, 2006).

Contudo, a despeito dessa divergência, a visão de um construto único tem sido predominante, e estudos empíricos têm apontado que os preditores da satisfação e da insatisfação não são distintos. Um exemplo seria o salário que era apontado na Teoria dos Dois Fatores como preditor da insatisfação no trabalho e que, posteriormente, foi apontado como preditor da satisfação (Ellickson, 2002) ou mesmo como seu componente (Siqueira, 1995; 2008). Dessa maneira, a avaliação do construto conta com um aspecto cognitivo derivado da interação do trabalhador com estímulos encontrados no ambiente laboral, em que cada pessoa elabora um significado particular (Judge \& Kammeyer-Mueller, 2012). Se por um lado, a satisfação no trabalho é considerada um construto unidimensional (Brayfield \& Rothe, 1951; Silva \& Ferreira, 2013) ou bidimensional (Locke, 1976), por outro, é também considerada em uma perspectiva multidimensional (Siqueira, 2008). 
Ancorada na perspectiva de Siqueira (2008), a satisfação no trabalho pode ser definida como uma decorrência de experiências e emoções vivenciadas no ambiente laboral que afetam a vida social, os vínculos afetivos e a saúde mental. Para mensurar o construto, a autora propôs a EST com o objetivo de avaliar o grau de contentamento do trabalhador frente a cinco fatores, nomeadamente: 1. satisfação com os colegas, correspondente a aspectos positivos mantidos com os pares; 2 . satisfação com o salário, representando a remuneração, em termos de quantidade de trabalho, capacidade profissional e custo de vida; 3 . satisfação com a chefia, voltada para a capacidade profissional do chefe e o seu interesse pelo trabalho dos subordinados; 4. satisfação com a natureza do trabalho, caracterizada pelo contentamento em relação às tarefas realizadas no ambiente laboral; e 5. satisfação com as promoções, que diz respeito aos critérios da empresa para promover pessoas e o número de promoções recebidas (Siqueira, 2008).

Esse modelo de satisfação no trabalho com cinco fatores correlacionados (Siqueira, 2008) tem sido amplamente utilizado ao longo dos anos (Coelho \& Faiad, 2012; Rueda, 2015; Silva et al., 2019) e é apontado como o instrumento mais utilizado em pesquisas brasileiras (Hora et al., 2018), o que salienta a necessidade de mais investigações sobre tal medida. Nesse sentido, destacamos a importância de investigar possibilidades variadas de estrutura fatorial para além do modelo correlacionado já proposto pela autora, tais como o modelo não correlacionado, hierárquico e bifatorial. A comparação de diferentes estruturas fatoriais contribui para investigações mais robustas sobre o fenômeno (Jeon et al., 2018; Reise et al., 2010).

Do ponto de vista do construto satisfação no trabalho, há respaldo na literatura para a testagem da estrutura hierárquica e de um fator geral independente das dimensões previstas no modelo de Siqueira, uma vez que a satisfação representa "uma totalização do quanto o indivíduo que trabalha vivencia experiências prazerosas no contexto das organizações" (Siqueira, 2008, p. 267). Nesse sentido, é possível que a satisfação vá além das dimensões colegas de trabalho, salário, chefia, natureza do trabalho e promoções, já que o construto se refere a uma experiência global do trabalhador no contexto laboral (Kunkel \& Vieira, 2012; Siqueira \& Gomide Jr., 2014; Spector, 2005).

Outro aspecto que precisa ser considerado acerca da medida é se ela se mantém invariante para diferentes amostras (Valentini et al., 2017). A testagem da invariância da EST deve levar em conta grupos que apresentam diferenças nas percepções do construto, nesse sentido, se for comprovada a invariância da medida, fica evidenciado que os diferentes escores obtidos pelo grupo não derivam de um mero erro de medida. Assim, tendo em vista que alguns estudos apontam diferenças nos níveis de satisfação laboral em função do tempo de trabalho, grau de escolaridade e sexo do participante (Kunkel \& Vieira, 2012; Lorenzo et al., 2020; Nascimento et al., 2019), optamos por investigar a invariância da medida tendo como balizas essas três variáveis.

Cumpre ainda destacar que o modelo teórico da satisfação pressupõe que cognições vão desenvolver afetos do trabalhador para com a empresa divididos em satisfação no trabalho, envolvimento com o trabalho e comprometimento organizacional afetivo, ocasionando um conjunto de pesquisas relacionando esses construtos no Brasil (Kunkel \& Vieira, 2012; Siqueira \& Gomide Jr., 2014; Siqueira \& Padovam, 2008) e no mundo (Ahmad et al., 2020; Mathieu et al., 2016). De fato, esses três construtos são considerados vínculos positivos com o trabalho e com a organização e são elementos relevantes para o bem-estar do trabalhador. Considerando que a inclusão de outros construtos para avaliar a validade convergente deve ser feita levando em conta evidências de correlações entre os construtos (Akremi et al., 2018; Johnson et al., 2012), optamos por testar a validade convergente da satisfação no trabalho com os construtos envolvimento com o trabalho e comprometimento organizacional afetivo.

Em face de tais considerações, o objetivo deste estudo foi identificar as propriedades relativas à Escala de Satisfação no Trabalho (Siqueira, 2008), em termos de estrutura fatorial, invariância da medida e validade convergente. A primeira hipótese é que modelos alternativos de ordem superior como os que serão comparados, a partir de análises confirmatórias, apresentam melhores ajustes aos dados empíricos. A segunda hipótese é que a Escala de Satisfação no Trabalho possui invariância da medida em termos de tempo de trabalho, grau de escolaridade e sexo. A terceira e última hipótese é que a satisfação no trabalho apresenta correlação positiva com envolvimento com o trabalho e com o comprometimento organizacional afetivo.

\section{Método}

\section{Participantes}

Participaram 733 trabalhadores brasileiros (46\% mulheres) do setor industrial e do setor terciário (prestação de serviços nas áreas de educação, serviço público e comércio). O perfil etário variou de 21 a 67 anos $(M=31,8$; $D P=8,4)$. A amostra foi de conveniência, abrangendo o estado da Bahia (69\%) e do Rio de Janeiro (31\%). Foram adotados como critérios de inclusão: 1. vínculo formal de trabalho na mesma empresa há pelo menos um ano, para que pudesse ser feita uma avaliação de satisfação laboral mais fidedigna; e 2. nível de escolaridade mínima de ensino médio completo, para o preenchimento do questionário.

A maioria dos participantes já trabalhavam há pelos menos dois anos na mesma empresa (47\%), e a escolaridade predominante foi nível superior completo (61\%). Houve predominância de trabalhadores solteiros (54\%), 
sem filhos (51\%), a média de carga horária de trabalho era de 35 horas semanais e a média salarial foi de $\mathrm{R} \$ 1.967,00$ $(D P=\mathrm{R} \$ 2.885,00)$, sendo que $50 \%$ dos pesquisados ganhavam até R\$1.034,00.

\section{Instrumentos}

Escala de Satisfação no Trabalho - EST (Siqueira, 2008). Utilizamos a versão reduzida composta por 15 itens subdivididos em cinco fatores (satisfação com os colegas, com o salário, com a chefia, com a natureza do trabalho e com as promoções). A medida apresenta bons indicadores de confiabilidade, no estudo original, o alfa de Cronbach variou de 0,77 a 0,90. Valores semelhantes $(0,79$ a 0,91$)$ foram obtidos por Rueda (2015), confirmando que a escala reduzida apresenta adequados indicadores de precisão. Foi utilizada uma escala de resposta do tipo Likert com cinco pontos (de totalmente satisfeito a totalmente insatisfeito). Um exemplo de item é: "No meu trabalho atual sinto-me... com o entendimento entre mim e meu chefe".

Escala de Envolvimento com o Trabalho (Lodahl \& Kejner, 1965, adaptado por Siqueira, 1995). É um instrumento unidimensional composto por cinco itens. O grau de confiabilidade da medida também foi satisfatório (alfa de Cronbach $=0,78$ ). Para a resposta, disponibilizamos uma escala Likert, de cinco pontos (variando de concordo plenamente a discordo plenamente). Um exemplo de item é: "Eu como, vivo e respiro meu trabalho".

Escala de Comprometimento Organizacional Afetivo (Siqueira, 1995). É um instrumento unidimensional composto por cinco itens e bom índice de confiabilidade (alfa de Cronbach=0,93). Utilizamos uma escala Likert de resposta, com cinco pontos, variando de concordo plenamente a discordo plenamente. Um exemplo de item é: "Sinto-me animado com a empresa".

Dados sociodemográficos. Desenvolvido especialmente para o presente estudo, o questionário sociodemográfico continha perguntas para caracterização da amostra e para a testagem da invariância da medida (sexo, escolaridade e tempo de trabalho).

\section{Procedimentos}

O projeto de pesquisa foi aprovado por um Comitê de Ética em Pesquisa. Todos os preceitos éticos exigidos foram considerados, tais como sigilo das respostas individuais, explicação sobre o objetivo da pesquisa, informação de que a pesquisa era voluntária e exigência de resposta do Termo de Consentimento Livre e Esclarecido. A coleta foi realizada de maneira on-line por meio de um link enviado por e-mail e por outras redes sociais, a partir da divulgação de quem já tinha respondido à pesquisa, caracterizando o emprego da técnica bola de neve. No caso de duas respostas de um mesmo e-mail, foi considerada apenas a última que foi enviada. Também foi feita uma checagem para verificar se todos os participantes que responderam ao questionário se enquadravam nos critérios de inclusão. A coleta de dados também foi feita de maneira presencial, com o questionário no formato impresso, em empresas que autorizaram a aplicação. Após a coleta presencial, os dados foram inseridos manualmente na plataforma virtual para evitar possíveis identificações. O tempo médio de resposta foi de 15 minutos.

\section{Análise de Dados}

As análises obtiveram o apoio do software $\mathrm{R}$ e foram realizadas em três fases. Inicialmente, foi feita análise fatorial confirmatória - AFC para verificar quais modelos se ajustavam aos dados empíricos, uma vez que a escala testada já tinha uma estrutura definida a priori em outros estudos e em amostras brasileiras (Franco et al., 2017). Todos os modelos comparados foram baseados na proposta teórica do instrumento, a saber: 1. modelo de cinco fatores correlacionados já proposto pela autora da escala; 2. modelo de cinco fatores não correlacionados; 3 . modelo hierárquico, que envolve as cinco variáveis latentes de primeiro nível e uma variável latente geral de segundo nível que explica diretamente as variáveis latentes de primeiro nível; e 4. modelo bifatorial, que envolve as cinco variáveis latentes de primeiro nível, que explica diretamente os itens, mais uma variável geral também de primeiro nível, que, de igual modo, explica os itens do instrumento.

A AFC foi realizada por meio do pacote lavaan (Rosseel, 2012) com o estimador Weighted Least Square Mean and Variance Ajusted (WLSMV), declarando-se os itens como variáveis ordinais. Os índices de ajuste absolutos adotados e os parâmetros estabelecidos foram: Root Mean-Square Error of Approximation (RMSEA <0,05); Comparative Fit Index (CFI>0,95); Normed Fit Index (NFI>0,90); e Non-normed Fit Index (NNFI>0,90) (Brown, 2006; Byrne, 2010). Também foi utilizado o cálculo da variância comum - Explained Common Variance (ECV) - para verificar a força do fator geral em modelos bifatoriais (Golino et al., 2015; Preacher \& MacCallum, 2003). Para a ECV, utiliza-se a seguinte equação:

$$
E C V=\frac{\sum \lambda^{2}{ }_{G e n}}{\left(\Sigma \lambda_{G e n}^{2}\right)+\left(\Sigma \lambda^{2}{ }_{F 1}\right)+\left(\Sigma \lambda^{2}{ }_{F 2}\right)+\ldots+\left(\sum \lambda_{F k}^{2}\right)}
$$

Na qual, $\lambda_{\text {Gen }}^{2}$ é o quadrado das cargas não padronizadas do fator geral. Já $\lambda_{F k}^{2}$ é o quadrado das cargas não padronizadas para cada uma das $\mathrm{k}$ variáveis latentes que, nesse caso, foram salário, colegas, chefia, promoções e natureza do trabalho. Já para comparação dos ajustes dos modelos testados, foi utilizado o teste de diferença de qui-quadrado escalonado (Satorra \& Bentler, 2010). 
Na segunda etapa da análise, foi testada a invariância da medida, por meio da técnica de análise fatorial confirmatória multigrupo - AFCMG. Para essa análise, foram consideradas as seguintes variáveis: 1. tempo de trabalho: com até dois anos na empresa e com três anos ou mais; 2. grau de escolaridade: ensino médio completo e nível superior completo; e 3. sexo: masculino e feminino. Tal análise engloba quatro etapas, a saber: invariância configural (em que medida a estrutura proposta é equivalente para todos os grupos?); a invariância métrica (em que medida as cargas fatoriais dos diferentes grupos se equivalem?); a invariância escalar (em que medida os interceptos são semelhantes para os grupos avaliados?); e a invariância da média da variável latente (em que medida os grupos apresentam igualdade de médias nos escores dos fatores?) (Damásio, 2013; Valentini et al., 2017). Os índices de ajuste utilizados foram os valores de CFI, RMSEA, delta CFI e delta RMSEA (variação nos diferentes modelos testados).

$\mathrm{Na}$ terceira e última fase da análise, foi realizada a testagem da validade convergente, referente às associações que o escore de uma escala apresenta com outras medidas externas (Valentini \& Damásio, 2017). Tal validade mostra o grau de semelhança entre os resultados de diferentes instrumentos para os quais se espera proximidade teórica. Assim, fundamentados no que é apontado pela literatura, analisamos a satisfação no trabalho com mais duas outras variáveis do comportamento organizacional, a saber: o envolvimento com o trabalho e o comprometimento organizacional afetivo. A análise foi feita a partir de correlação entre as variáveis, sendo empregado o coeficiente de correlação de Spearman, em função de os itens terem sido declarados como ordinais. Para a avaliação da magnitude das correlações, adotou-se o critério de Miles e Shevlin (2001) categorizado em correlações baixas (até 0,30 ), correlações moderadas (de 0,30 a 0,50 ) e correlações elevadas (acima de 0,50 ).

\section{Resultados}

$\mathrm{Na}$ primeira fase de análise, foram testados quatro modelos alternativos para a estrutura da EST (Siqueira, 2008): o primeiro que pressupunha dimensões não correlacionadas, o segundo que seria o próprio modelo de Siqueira (2008) com estrutura de cinco fatores correlacionados, o terceiro um modelo hierárquico e o quarto um modelo bifatorial. O modelo de cinco fatores não correlacionados não se ajustou aos dados empíricos, indicando que a estrutura da medida contempla fatores associados $\left(\chi^{2}=5.253,5 ; \mathrm{CFI}=0,43 ; \mathrm{NFI}=0,48\right.$; $\mathrm{NNFI}=0,33$; RMSEA $=0,28)$.

O segundo modelo testado foi o de cinco fatores correlacionados (satisfação com os colegas, satisfação com o salário, satisfação com a chefia, satisfação com a natureza do trabalho e satisfação com as promoções). Os resultados mostraram qui-quadrado significativo $\left(\chi^{2}=35,69\right)$, mas os demais indicadores apontaram para um modelo de excelente ajuste aos dados empíricos $(\mathrm{CFI}=1,00 ; \mathrm{NFI}=0,99 ; \mathrm{NNFI}=1,00 ; \mathrm{RMSEA}=0,00)$, o que corrobora com a proposta original da EST (Siqueira, 2008).

O terceiro modelo foi o hierárquico com cinco variáveis latentes de primeiro nível da satisfação no trabalho e uma variável latente geral de segundo nível que explica diretamente as cinco variáveis latentes de primeiro nível. Tal modelo se ajustou aos dados empíricos com qui-quadrado significativo $\left(\chi^{2}=109,89\right)$. Os resultados apontam para a existência de um fator geral que explica a satisfação com os colegas, com o salário, com a chefia, com a natureza do trabalho e com as promoções ( $\mathrm{CFI}=0,99$; $\mathrm{NFI}=0,98 ; \mathrm{NNFI}=0,99 ; \mathrm{RMSEA}=0,03) . \mathrm{O}$ modelo hierárquico salienta, então, que uma variável latente é adequada para explicar as variáveis latentes de primeiro nível, nesse caso, das cinco dimensões concebidas por Siqueira (2008).

O quarto e último modelo testado foi o bifatorial, com as cinco variáveis latentes de primeiro nível (colegas, salário, chefia, natureza do trabalho e promoções) explicando diretamente os 15 itens do instrumento, e mais uma variável geral, também de primeiro nível, explicando todos os itens do instrumento. O qui-quadrado, assim como nos demais modelos foi significativo $\left(\chi^{2}=100,59\right)$ e os resultados apontaram que tal modelo também obteve ótimo ajuste aos dados empíricos ( $\mathrm{CFI}=0,99$; $\mathrm{NFI}=0,98 ; \mathrm{NNFI}=0,99 ; \mathrm{RMSEA}=0,02$ ).

De maneira sintética, os resultados permitem indicar diferentes possibilidades de estrutura para a Escala de Satisfação no Trabalho, quer seja o modelo de cinco fatores correlacionados, o hierárquico e o bifatorial. Este último além das cinco variáveis latentes de primeiro nível, indica que o fator geral tem uma contribuição direta para a explicação dos itens da satisfação no trabalho, que são simultaneamente explicados pelas dimensões específicas da satisfação no trabalho. $\mathrm{O}$ uso desse modelo gera uma situação rica do ponto de vista psicométrico, pois a variável geral compete com as cinco variáveis específicas da satisfação - colegas, salário, chefia, natureza do trabalho e promoções - na explicação da variância dos itens. A Figura 1 apresenta as cargas padronizadas do modelo bifatorial da satisfação no trabalho.

Optamos por seguir nas análises seguintes com o modelo bifatorial, porque ele apresentou uma possibilidade rica de explicação dos resultados, uma vez que a ECV do modelo bifatorial resultou que o fator geral de primeiro nível explicou $77 \%$ da variância comum dos itens, e os 33\% restantes da variância foram explicados pelos cinco fatores específicos da satisfação no trabalho. Assim, foi testado se os parâmetros do modelo bifatorial eram equivalentes para tempo de trabalho, escolaridade e sexo. Os resultados apontam invariância configural, métrica e escalar, só não tendo sido obtida a invariância da média da variável latente, o que pode ser explicado 
em função de a satisfação no trabalho depender, dentre outros, das características dos respondentes. A Tabela 1 apresenta os valores obtidos em cada tipo de análise de invariância testada em seus respectivos grupos, bem como os resultados encontrados nos índices de ajustes considerados.

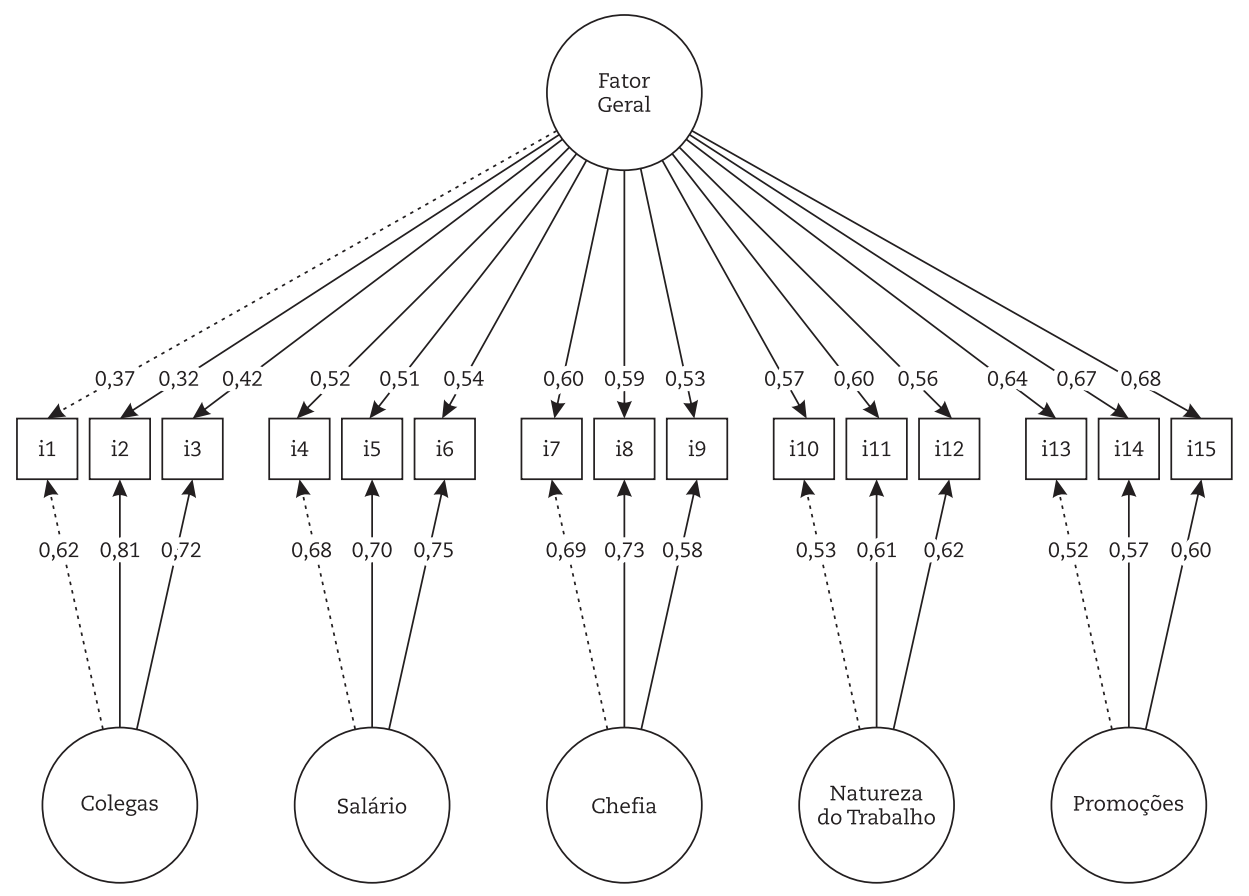

Figura 1. Cargas Padronizadas do Modelo Bifatorial da Satisfação no Trabalho

Tabela 1

Análise de Invariância do Modelo Bifatorial da Satisfação no Trabalho

\begin{tabular}{lllccc}
\hline \multicolumn{1}{c}{ Grupo } & \multicolumn{1}{c}{ Tipo } & CFI & RMSEA & Delta CFI & \multicolumn{2}{c}{ Delta RMSEA } \\
\hline \multirow{3}{*}{ Tempo de Trabalho } & Configural & 0,969 & 0,041 & NA & NA \\
& + Cargas & 0,985 & 0,026 & 0,016 & 0,014 \\
& + Interceptos & 0,984 & 0,026 & 0,001 & 0,000 \\
& + Médias & 0,984 & 0,026 & 0,000 & 0,001 \\
& Configural & 0,968 & 0,041 & NA & NA \\
Escolaridade & + Cargas & 0,983 & 0,028 & 0,014 & 0,013 \\
& + Interceptos & 0,982 & 0,028 & 0,001 & 0,000 \\
& + Médias & 0,972 & 0,034 & 0,010 & 0,006 \\
& & & & NA & NA \\
Sexo & Configural & 0,970 & 0,040 & 0,003 & 0,001 \\
& + Cargas & 0,967 & 0,039 & 0,001 & 0,001 \\
& + Interceptos & 0,966 & 0,039 & 0,011 & 0,005 \\
\hline
\end{tabular}

Os resultados indicam que, para todos os modelos testados, o CFI apresentou valores acima de 0,95 e que o RMSEA foi inferior a 0,05 , apontando para a parcimônia dos modelos testados (Brown, 2006; Byrne, 2010). Portanto, o modelo bifatorial é estatisticamente adequado para avaliar a satisfação e do ponto de vista da análise de invariância, a EST apresenta a mesma estrutura configural, equivalência de cargas fatoriais e de interceptos para os diferentes grupos testados. Esses resultados autorizam a utilização da escala para diferentes públicos-alvo em termos de tempo de trabalho na mesma empresa, grau de escolaridade e sexo, e ampliam a possibilidade de 
se utilizar escores computados, considerando a estrutura bifatorial da escala. Esse resultado aponta para a necessidade de se alterar a forma como os escores da escala são gerados para o seu uso em pesquisas e atividades de levantamento organizacional. Uma das formas de se gerar escores que reflitam a estrutura fatorial da escala é por meio do cômputo dos escores fatoriais da análise fatorial Full Information Maximum Likelihood - FIML (Kamata \& Bauer, 2008), que permite estimar a estrutura bifatorial seguindo tanto um modelo fatorial quanto utilizando a teoria de resposta ao item (que no caso da FIML são equivalentes).

$\mathrm{Na}$ terceira e última fase da análise, foi realizada a correlação da EST com as escalas de envolvimento com o trabalho e de comprometimento organizacional afetivo, por meio do coeficiente de correlação de Spearman. Os resultados encontrados confirmam que os fatores apresentam correlações significativas e positivas, o que denota, portanto, variância comum entre os construtos (Tabela 2).

Tabela 2

Correlação de Spearman das Escalas de Satisfação no Trabalho, Envolvimento com o Trabalho e Comprometimento Organizacional Afetivo

\begin{tabular}{lcc}
\hline \multicolumn{1}{c}{ Satisfação } & Envolvimento & Comprometimento \\
\hline Satisfação com os colegas de trabalho & 0,19 & 0,31 \\
Satisfação com a chefia & 0,30 & 0,55 \\
Satisfação com as promoções & 0,43 & 0,53 \\
Satisfação com o salário & 0,33 & 0,43 \\
Satisfação com a natureza do trabalho & 0,45 & 0,53 \\
Fator geral de satisfação (modelo bifatorial) & 0,49 & 0,67 \\
\hline
\end{tabular}

Do conjunto das 12 correlações testadas, quase todas apresentaram magnitude moderada ou elevada $(0,30$ a 0,50 ) à exceção, apenas, da dimensão satisfação com os colegas que teve correlação fraca $(0,19)$ com o envolvimento com o trabalho. Assim, confirma-se, a validade convergente entre as escalas propostas. As correlações mais elevadas foram encontradas na correlação do fator geral da satisfação no trabalho do modelo bifatorial com o comprometimento organizacional afetivo $(0,67)$ e com o envolvimento no trabalho $(0,49)$.

\section{Discussão}

O objetivo deste estudo foi identificar as propriedades relativas à Escala de Satisfação no Trabalho (Siqueira, 2008), em termos de estrutura fatorial, invariância da medida e validade convergente. A escolha da EST (Siqueira, 2008) deveu-se, não apenas por ser a mais utilizada em pesquisas brasileiras (Hora et al., 2018), mas também por permitir uma mensuração mais detalhada do construto, ao contrário de modelos unifatoriais, como o de Silva e Ferreira (2013) ou bifatoriais, como o de Locke (1976). Nesse sentido, ao usar o modelo de cinco dimensões (satisfação com colegas de trabalho, salário, chefia, natureza do trabalho e promoções), é possível conhecer melhor em que aspectos o trabalhador encontra-se ou não satisfeito com o seu trabalho.

Além do modelo de cinco dimensões correlacionadas que já havia sido apresentado anteriormente por Siqueira (2008), os resultados evidenciaram que o modelo hierárquico e o modelo bifatorial apresentaram índices adequados. Assim, confirmamos a primeira hipótese deste estudo de que modelos alternativos de ordem superior apresentam bons ajustes aos dados empíricos. Embora o modelo correlacionado (Siqueira, 2008) e o modelo hierárquico também tenham se ajustado aos dados empíricos, no modelo bifatorial, a variável geral compete com as variáveis específicas na explicação da variância dos itens do instrumento, o que gera um resultado psicométrico mais robusto (Jeon et al., 2018; Reise et al., 2010). Tal resultado também tem respaldo teórico, uma vez que a satisfação é entendida como o resultado de uma totalização das experiências prazerosas que os indivíduos vivenciam no trabalho, ou seja, o construto se refere a uma experiência global do trabalhador no contexto laboral (Kunkel \& Vieira, 2012; Siqueira, 2008; Spector, 2005).

Nesse sentido, evidenciamos que o modelo de Siqueira (2008) oferece diferentes possibilidades para a investigação da satisfação dos trabalhadores e apresenta bons indicadores, reiterando estudos prévios que testaram essa medida (Coelho \& Faiad, 2012; Rueda, 2015; Silva et al., 2019). Além disso, confirmando a segunda hipótese deste estudo, destacamos que o modelo bifatorial de satisfação no trabalho é invariante, exceto para as médias das variáveis latentes, o que permite indicar um amplo uso da EST, independentemente do tempo de trabalho, grau de escolaridade e sexo dos participantes.

Acerca da testagem do modelo bifatorial, outros pesquisadores já sinalizavam para a possibilidade desse tipo de modelo em fenômenos psicológicos (Jeon et al., 2018; Reise et al., 2010). Assim, estudos que realizaram comparações entre modelos estruturais indicam que, em alguns 
casos, a aplicação de modelos bifatoriais oferece informações mais ricas sobre os fenômenos, uma vez que o modelo bifatorial permite a estimação de uma variável latente geral e o controle da variância que emerge de fatores específicos (Costa \& Faria, 2020; Golino et al., 2016). Dessa forma, é uma estrutura indicada quando se quer investigar se os itens de um instrumento avaliam, para além das variáveis latentes de seus fatores, uma variável latente de fator único comum (Jeon et al., 2018; Reise et al., 2010).

Diante dos resultados obtidos neste estudo, o modelo bifatorial pode ser empregado nas pesquisas sobre satisfação no trabalho a depender dos objetivos e hipóteses a serem testadas em cada estudo. Assim, utilizar o modelo de cinco fatores de Siqueira (2008) mais um fator geral de explicação tem uma contribuição direta para a investigação da satisfação dos trabalhadores brasileiros. Portanto, quando o objetivo do pesquisador for identificar o grau de satisfação dos trabalhadores, o modelo bifatorial é o mais conveniente.

Além de investigar a estrutura fatorial e a invariância da medida, também testamos a validade convergente da satisfação no trabalho com outras variáveis do comportamento organizacional. Os resultados apontaram que a terceira hipótese também foi confirmada, ao passo que a EST teve correlação positiva de moderada a elevada com as escalas de envolvimento com o trabalho e de comprometimento organizacional afetivo. As correlações mais elevadas foram encontradas na correlação do fator geral da satisfação no trabalho presente no modelo bifatorial. Diante disso, evidenciamos a sua validade convergente como já apontado por outros pesquisadores do tema (Kunkel \& Vieira, 2012; Siqueira \& Gomide Jr., 2014; Siqueira \& Padovam, 2008).

Assim é que os resultados do presente estudo, tomados em conjunto, permitem um uso mais apropriado da medida de satisfação no trabalho, o que contribui para melhor compreender a natureza do fenômeno investigado. Embora o uso de construções multidimensionais de ordem superior na área de POT e nas literaturas comportamentais ter se tornando comum (Jeon et al., 2018; Johnson et al., 2012), estudos sobre a medida da satisfação no trabalho em brasileiros ainda carece na literatura. Nesse sentido, as organizações brasileiras podem se valer dos resultados aqui apresentados para mensurar o grau de satisfação de seus trabalhadores e adotar medidas mais robustas que resultem em estratégias para aumentar os níveis de satisfação em todas as dimensões consideradas. A medida também pode ser utilizada para identificar setores com mais e menos problemas, além de modelos preditivos que apontem as variáveis que levam os funcionários a se sentirem mais satisfeitos. Esse tipo de pesquisa pode ser útil, então, para a elaboração de estratégias de intervenção relacionadas ao comportamento organizacional positivo dos trabalhadores.

Uma das limitações deste estudo foi ter testado apenas um instrumento de satisfação no trabalho. A testagem de outros instrumentos, que seguem modelos teóricos distintos pode ser interessante para oferecer aos pesquisadores outras possibilidades para mensuração da satisfação. No entanto, é preciso considerar que o instrumento de Siqueira (2008) é o mais utilizado em pesquisas brasileiras e, nesse sentido, tende a contribuir com grande parte das pesquisas nacionais sobre o tema.

Apesar de este estudo apresentar testes de invariância da medida para tempo de trabalho, grau de escolaridade e sexo, que representam um avanço em relação a estudos anteriores, estudos futuros podem expandir a avaliação de invariância da medida para outras variáveis, como tamanho da organização e tipo de profissão. Seria útil ainda, como agenda de pesquisa, a mensuração da estabilidade temporal da medida e uma ampliação da amostra para estados de outras regiões do Brasil. Sugere-se também a construção de uma medida pictórica que permita uma aplicação mais rápida e, possivelmente, menos ancorada em expectativas de resposta socialmente desejáveis.

Cumpre ressaltar, por fim, que o estudo se reveste de importância teórica e prática em função de ter testado a escala de satisfação mais utilizada em estudos nacionais. A avaliação de diferentes estruturas para a medida e a indicação de um modelo estrutural robusto para análise do construto pode, portanto, contribuir para o avanço das pesquisas sobre o tema. O fato de concluir que a medida é convergente com construtos semelhantes e invariante aos diferentes grupos propostos aumenta as possibilidades de uso da medida e da comparação de resultados para segmentos distintos.

\section{Agradecimentos}

Agradecemos a todos os participantes do presente estudo que investiram tempo respondendo o questionário de pesquisa.

\section{Financiamento}

Todas as fontes de financiamento para elaboração e produção do estudo (coleta, análise e interpretação dos dados, bem como, escrita dos resultados no presente no manuscrito) foram fornecidas pelo projeto de pesquisa - Coordenação de Aperfeiçoamento de Pessoal de Nível Superior (CAPES), código de financiamento 001; Conselho Nacional de Desenvolvimento Científico e Tecnológico (CNPq), código de financiamento 312109/2017-2; e Fundação de Amparo à Pesquisa do Estado do Rio de Janeiro (FAPERJ), Processo $n^{\circ}$ E-26/202.913/2018.

\section{Contribuições dos autores}

Todos os autores participaram da elaboração do manuscrito. Especificamente, o(s) autor(es) Luara Carvalho, Hudson Golino e Luciana Mourão participaram da redação inicial do estudo - conceitualização, investigação, 
visualização, o(s) autor(es) Luara Carvalho e Hudson Golino participaram da análise dos dados, e o(s) autor(es) Luara Carvalho e Luciana Mourão participaram da redação final do trabalho - revisão e edição.

\section{Disponibilidade dos dados e materiais}

Todos os dados e sintaxes gerados e analisados durante esta pesquisa serão tratados com total sigilo devido às exigências do Comitê de Ética em Pesquisa em Seres Humanos. Porém, o conjunto de dados e sintaxes que apoiam as conclusões deste artigo estão disponíveis mediante razoável solicitação ao autor principal do estudo.

\section{Conflito de interesses}

Os autores declaram que não há conflitos de interesses.

\section{Referências}

Ahmad, K., Alwee, S., Yusoff, Z., Osman, S., \& Tuah, S. (2020). The Association between ethical decision-making, job satisfaction, organisational commitment and selected demographic variables. Malaysian Management Journal, 7(2), 1-11. http://e-journal.uum.edu. my/index.php/mmj/article/view/8749

Akremi, A., Gond, J., Swaen, V., Roeck, K., \& Igalens, J. (2018). How do employees perceive corporate responsibility? Development and validation of a multidimensional corporate stakeholder responsibility scale. Journal of Management, 44(2), 619-657. https://doi. org $/ 10.1177 / 0149206315569311$

Begley, T., \& Czajka, J. (1993). Panel analysis of the moderating effects of commitment on job satisfaction, intent to quit, and health following organizational change. Journal of Applied Psychology, 78(4), 552-556. https://doi.org/10.1037/0021-9010.78.4.552

Borges-Andrade, J., \& Zanelli, J. (2014). Psicologia e produção do conhecimento em organizações e trabalho. Em J. Zanelli, J. BorgesAndrade, \& A. Bastos, (Eds.), Psicologia, Organizações e Trabalho no Brasil (pp. 583-608). Artmed.

Brayfield, A., \& Rothe, H. (1951). An index of job satisfaction. Journal of Applied Psychology, 35, 307-311. https://doi.org/10.1037/h0055617

Brown, T. (2006). Confirmatory factor analysis for applied research. Guilford.

Byrne, B. (2010). Structural equation modeling with AMOS: Basic concepts, applications, and programming (2nd ed.). Routledge, Taylor \& Francis.

Coelho F., \& Faiad, C. (2012). Evidências de validade da Escala de Satisfação no trabalho. Avaliação Psicológica, 11(1), 111-121. http://redalyc. org/articulo.oa?id $=335027499011$

Costa, A., \& Faria, L. (2020). Construção e Validação da Escala de Teorias Implícitas de Inteligência Emocional (TIIE) no Contexto Escolar. Revista Iberoamericana de Diagnóstico y Evaluación Psicológica, 1(54), 57-68. https://doi.org/10.21865/RIDEP54.1.05

Damásio, B. (2013). Contribuições da Análise Fatorial Confirmatória Multigrupo (AFCMG) na avaliação de invariância de instrumentos psicométricos. Psico-USF, 18(2), 211-220. https://doi.org/10.1590/S1413-82712013000200005

Ellickson, M. C. (2002). Determinants of job satisfaction of municipal government employees. Public Personnel Management, 31 (3), $343-358$. https://doi.org/10.1177/009102600203100307

Farina, L., Rodrigues, G., Fagundes, N., Carafini, T., Moreira, L., Machado, W., \& Hutz, C. (2019). Flow e Engajamento no Trabalho: Associações com Recursos Individuais e Contexto Laboral. Avaliação Psicológica, 18(4), 362-371. https://doi.org/10.15689/ ap.2019.1804.18813.04

Franco, V., Valentini, F., \& Iglesias, F. (2017). Introdução à análise fatorial confirmatória. Em B. Damásio \& J. Borsa. (Eds.), Instrumentos Psicológicos: Manual de desenvolvimento (pp. 295-322). Vetor.

Golino, H., Carvalho, L., Silva, E., Oliveira, E., Musse, S., \& Silva, B. (2016). Revisão do Inventário de Ciúme Romântico: Uma análise via modelo de Rasch. Avaliação Psicológica, 15(3), 285-297. https://doi.org/10.15689/ap.2016.1503.01

Golino, H., \& Gomes, C., Amantes, A., \& Coelho, G. (2015). Psicometria Contemporânea: Compreendendo os Modelos Rasch. Casa do Psicólogo/ Pearson.

Hackman, J. R., \& Oldham, G. R. (1975). Development of the Job Survey. Journal of Applied Psychology, 60(2), 159-170. https://doi. org $/ 10.1037 / \mathrm{h} 0076546$

Hall, D., Yip, J., \& Doiron, K. (2018). Protean careers at work: Self-direction and values orientation in psychological success. Annual Review of Organizational Psychology and Organizational Behavior, 5, 129-156. https://doi.org/10.1146/annurev-orgpsych-032117-104631

Herzberg, F., Mausner, B., \& Snyderman, B. (1959). The motivation to work. Wiley.

Hora, G., Ribas Júnior, R., \& Souza, M. (2018). Estado da arte das medidas em satisfação no trabalho: Uma revisão sistemática. Trends in Psychology, 26(2), 971-986. https://doi.org/ 10.9788/tp2018.2-16pt

International Test Comission (2018). International Test Commission Guidelines for Translating and Adapting Tests. International Journal of Testing, 18(2), 101-134. https://doi.org/10.1080/15305058.2017.1398166

Jeon, M., Rijmen, F., \& Rabe-Hesketh, S. (2018). CFA Models with a General Factor and Multiple Sets of Secondary Factors. Psychometrika, 83(4), 785-808. https://doi.org/10.1007/s11336-018-9633-x

Judge, T., Parker, S., Colbert, A., Heller, D., \& Iles, R. (2001). Job Satisfaction: A Cross-cultural Review. In N. Anderson, D. Ones, H. Sinangil, \& C. Viswesvaran (Eds.), Handbook of Industrial, Work \& Organizational Psychology (pp. 25-52). Sage.

Judge, T., \& Kammeyer-Mueller, J. (2012). Job Attitudes. Annual Review of Psychology, 63, 341-367. https://doi.org/10.1146/annurevpsych-120710-100511

Johnson, R., Rosen, C., Chang, C., Djurdjevic, E., \& Taing, M. (2012). Recommendations for improving the construct clarity of higherorder multidimensional constructs. Human Resource Management Review, 22, 62-72. https://doi.org/10.1016/j.hrmr.2011.11.006

Kamata, A., \& Bauer, D. J. (2008). A note on the relation between factor analytic and item response theory models. Structural Equation Modeling: A Multidisciplinary Journal, 15(1), 136-153. https://doi.org/10.1080/10705510701758406

Kunkel, F., \& Vieira, K. (2012). Bem-estar no Trabalho: Um estudo junto aos servidores públicos da prefeitura municipal de cerro largo, RS. Gestão \& Regionalidade, 28(83). https://doi.org/10.13037/gr.vol28n83.1789

Locke, E. (1976). The nature and causes of job satisfaction. In M. Dunnette (Eds.), Handbook of Industrial and Organizational Psychology (pp. 1297-1350). Rand McNally College Publishing Company.

Lodahl, T., \& Kejner, M. (1965). The definition and measurement of job involvement. Journal of Applied Psychology, 49, 23-33. https://doi. org/10.1037/h0021692 
Lorenzo, S., Alves, A., \& Silva, N. (2020). Burnout e satisfação no trabalho em professores do ensino infantil. Brazilian Journal of Development, 6(5), 26937-26950. https://doi.org/10.34117/bjdv6n5-226

Marqueze, E., \& Moreno, C. (2005). Satisfação no trabalho - Uma breve revisão. Revista Brasileira de Saúde Ocupacional, 30 (112), 69-79. https://doi.org/10.1590/S0303-76572005000200007

Martins, M., \& Santos, G. (2006). Adaptação e validação de construto da Escala de Satisfação no Trabalho. Psico-USF, 11(2), 195-205. https:// doi.org/10.1590/S1413-82712006000200008

Mathieu, C., Fabi, B., Lacoursière, R., \& Raymond, L. (2016). The role of supervisory behavior, job satisfaction and organizational commitment on employee turnover. Journal of Management \& Organization, 22(1), 113-129. https://doi.org/10.1017/jmo.2015.25

Miles, J., \& Shevlin, M. (2001). Applying regression and correlation: A guide for students and researchers. Sage Publications.

Moura, A., Bernardes, A., Balsanelli, A., Dessotte, C., Gabriel, C., \& Zanetti, A. (2020). Leadership and job satisfaction in the Mobile Emergency Care Service context. Revista Latino-Americana de Enfermagem, 28, e33260. https://doi.org/10.1560/1518-8345.3455.3260

Mourão, L., Bastos, A., \& Oliveira, R. (2016). The saying and the doing in research on WOP. Revista Psicologia Organizações e Trabalho, 16(4), 333-339. https://doi.org/10.17652/rpot/2016.4.12575

Nascimento, R., Farias, G., Pereira, M., Both, J., \& Folle, A. (2019). Satisfação no trabalho: Associação com características pessoais de professores de Educação Física. Pensar en Movimiento, 17(2), 24-39. https://doi.org/10.15517/pensarmov.v17i2.34947

O'Reilly, C. (1991). Organizational Behavior: Where We'Ve Been, Where Were Going. Annual Review of Psychology, 42, 427-458. https://doi. org/10.1146/annurev.ps.42.020191.002235

Preacher, K., \& MacCallum, R. (2003). Repairing Tom Swift's electric factor analysis machine. Understanding Statistics: Statistical issues in psychology, education, and the social sciences, 2(1), 13-32. https://doi.org/10.1207/S15328031US0201 02

Reise, S., Moore, T., \& Haviland, M. (2010). Bifactor models and rotations: Exploring the extent to which multidimensional data yield univocal scale scores. Journal of Personality Assessment, 92(6), 544-559. https://doi.org/10.1080/00223891.2010.496477

Rodrigues, A., \& Carvalho-Freitas, M. (2016). Theoretical fragmentation: Origins and repercussions in Work and Organizational Psychology. Revista Psicologia, Organizações e Trabalho, 16(4), 310-315. https://doi.org/10.17652/rpot/2016.4.12630

Rosseel, Y. (2012). Lavaan: An R Package for Structural Equation Modeling. Journal of Statistical Software, 48(2), 1-36. https://link.springer. com/article/10.1007\%2FBF03396886

Rueda, F. (2015). Análise fatorial confirmatória da Escala de Satisfação no Trabalho nas versões de 25 e 15 itens. Revista Psicologia Organizações e Trabalho, 15(1), 82-88. https://doi.org/10.17652/rpot/2015.1.436

Satorra, A., \& Bentler, P. (2010). Ensuring Positiveness of the Scaled Difference Chisquare Test Statistic. Psychometrika, 75, 243-248. https:// doi.org/10.1007/S11336-009-9135-Y

Silva, C., \& Ferreira, M. (2013). Dimensões e indicadores da qualidade de vida e do bem-estar no trabalho. Psicologia: Teoria e Pesquisa, 29(3), 331-339. https://doi.org/10.1590/S0102-37722013000300011

Silva, E., Paulino, R., Silva, F., \& Oliveira, C. (2019). A influência do estilo de liderança na satisfação do trabalho: estudo em uma unidade de ensino particular no município de Bananeiras-PB. Qualitas Revista Eletrônica, 20(2), 89-114. https://doi.org/10.18391/req.v20i2.3985

Siqueira, M. (1995). Antecedentes de comportamentos de cidadania organizacional: Análise de um modelo pós-cognitivo [Unpublished doctoral dissertation]. Universidade de Brasília.

Siqueira, M. (2008). Satisfação no trabalho. Em M. Siqueira (Eds.), Medidas do comportamento organizacional: Ferramentas de diagnóstico e gestão (pp. 265-274). Artmed.

Siqueira, M., \& Padovam, V. (2008). Bases teóricas de bem-estar subjetivo, bem-estar psicológico e bem-estar no trabalho. Psicologia: Teoria e Pesquisa, 24(2), 201-209. https://doi.org/10.1590/S0102-37722008000200010

Siqueira, M., \& Gomide Jr., S. (2014). Vínculos do indivíduo com o trabalho e com a organização. Em J. Zanelli, J. Borges-Andrade, \& A. Bastos, (Eds.), Psicologia, Organizações e Trabalho no Brasil (pp. 316-348). Artmed.

Spector, P. E. (2005). Introduction: The dispositional approach to job satisfaction. Journal of Organizational Behavior, 26(1), 57. https://doi. org/10.1002/job.297

Staw, B. (1984). Organizational behavior: a review and reformulation of the field's outcome variables. Annual Review of Psychology, 35, 627666. https://doi.org/10.1146/annurev.ps.35.020184.003211

Valentini, F., \& Damásio, B. (2017). Variância média extraída e confiabilidade composta: Indicadores de precisão. Psicologia: teoria e pesquisa, 32(2), 1-7. https://doi.org/10.1590/0102-3772e322225

Valentini, F., Franco, V., \& Iglesias, F. (2017). Introdução à análise de invariância: Influência de variáveis categóricas e intervalares na parametrização dos itens. Em B. Damásio \& J. Borsa. (Eds.), Instrumentos Psicológicos: Manual de desenvolvimento (pp. 347-376). Vetor.

van Vianen, A. (2018). Person-Environment Fit: A Review of Its Basic Tenets. Annual Review of Psychology and Organizational Behavior, 5(1), 75-101. https://doi.org/10.1146/annurev-orgpsych-032117-104702

Yasin, Y., Kerr, M., Wong, C., \& Bélanger, C. (2020). Factors affecting nurses' job satisfaction in rural and urban acute care settings: A PRISMA systematic review. Journal of Advanced Nursing, 76(4), 963-979. https://doi.org/10.1111/jan.14293

\section{Sobre os autores}

Luara Carvalho é psicóloga, mestre e doutoranda em Psicologia pela Universidade Salgado de Oliveira (UNIVERSO).

Hudson Golino é psicólogo e doutor em Psicologia pela Universidade Federal de Minas Gerais (UFMG). Atualmente é Professor Assistente de Métodos Quantitativos no Departamento de Psicologia da University of Virginia.

Luciana Mourão é doutora em Psicologia pela Universidade de Brasília (UnB). Atualmente é Professora na Pós-Graduação Stricto Sensu em Psicologia da Universidade Salgado de Oliveira (UNIVERSO) e na Universidade Estadual do Rio de Janeiro (UERJ).

\section{Como citar este artigo}

Carvalho, L., Golino, H., \& Mourão, L. (2021). Evidências Adicionais de Validade da Escala de Satisfação no Trabalho. Avaliação Psicológica, 20(1), 61-70. http://dx.doi.org/10.15689/ap.2021.2001.17426.07 